\title{
Demographic Analysis of Breeding Structure in Japanese Thoroughbred Population
}

\author{
Jun YAMASHITA ${ }^{1}$, Hironori OKI ${ }^{2}$, Telhisa HASEGAWA ${ }^{2}$, Takeshi HONDA ${ }^{3}$ and \\ Tetsuro NOMURA ${ }^{4 *}$ \\ ${ }^{1}$ Department of Biotechnology, Faculty of Engineering, Kyoto Sangyo University, Kita-ku, Kyoto 603-8555, \\ ${ }^{2}$ Equine Research Institute, Japan Racing Association, 321-4 Tokami-cho, Utsunomiya, Tochigi 320-0856, \\ ${ }^{3}$ Food Resources Education and Research Center, Graduate School of Agricultural Science, Kobe University, \\ 1348 Uzurano-cho, Kasai, Hyogo 675-2103, ${ }^{4}$ Department of Bioresource and Environmental Sciences, Faculty \\ of Life Sciences, Kyoto Sangyo University, Kita-ku, Kyoto 603-8555, Japan
}

To investigate the breeding structure in the Japanese Thoroughbred population, we applied a demographic analysis to the populations of foals produced from 1978 to 2005. The migration rate estimated from the proportion of foals produced by imported breeding horses was around $40 \%$ over the investigated period. After early 1990s, the migration rate through stallions imported from USA sharply increased. The average generation interval was within range of 10.5-11.5 years. The longer generation interval of Thoroughbred was considered to be a reflection of the fact that Thoroughbred horses begin breeding only after completing their performance in races. After the peak of 729 in 1993, the number of sires of foals progressively declined to 358 in 2005. Although the coefficient of variation of the progeny number of sires was within range of 1.0-1.2 until early 1990s, it gradually increased and reached the value of 1.6-1.7 in recent years. The effective number of sires consistently decreased after the peak of 302.6 in 1992, and reached 120-130 in recent years, which is 25-30\% of the actual number of sires. In parallel, the demographic estimate of the effective population size declined after early 1990s. The main cause of the observed change in the breeding structure was inferred to be the intensive use of a limited number of stallions for breeding.

Key words: breeding structure, demographic analysis, effective population size, Thoroughbred
J. Equine Sci.

Vol. 21, No. 2

pp. 11-16, 2010
The first stud book for Thoroughbred horses was published in 1791, compiling earlier sources of pedigree information for a small number of horses of either imported or local English origin [2]. The current population expands to more than 300,000 world-wide, and Thoroughbred racing constitutes a huge leisure industry. Because this breed has been effectively closed to other horse breeds since its foundation, there is continuing concern that the reduced genetic diversity may be limiting genetic progress in performance [1, 2, 4, 12]. In fact, Cunningham [1] revealed that winning times in classic

This article was accepted April 14, 2010

*Corresponding author. e-mail: nomurat@cc.kyoto-su.ac.jp
Thoroughbred races in England showed no improvements during the recent decades.

We attempted a series of studies to investigate the breeding structure and evaluate genetic diversity in the Japanese Thoroughbred population by demographic and pedigree analysis. In this paper, we report results of demographic analysis of the breeding structure, which provide basic information for the succeeding pedigree analysis. The results are also compared with those for a major beef breed in Japan, the Japanese Black cattle, of which breeding structure has been intensively studied by two of the authors of this report $[5,6,9,10]$. While reproduction in Thoroughbred is strictly limited to natural service, almost all the progeny of the Japanese Black cattle are produced by artificial insemination with frozen semen. The comparison of 
Table 1. The number of foals by sex, used for demographic analysis

\begin{tabular}{|c|c|c|c|c|c|c|c|}
\hline Year & Male & Female & Gelding & Year & Male & Female & Gelding \\
\hline 1978 & 3,628 & 3,551 & 0 & 1992 & 5,205 & 5,240 & 0 \\
\hline 1979 & 3,554 & 3,567 & 0 & 1993 & 5,192 & 5,007 & 0 \\
\hline 1980 & 3,602 & 3,613 & 0 & 1994 & 4,977 & 4,860 & 0 \\
\hline 1981 & 3,609 & 3,685 & 0 & 1995 & 4,667 & 4,488 & 0 \\
\hline 1982 & 3,732 & 3,756 & 0 & 1996 & 4,567 & 4,410 & 0 \\
\hline 1983 & 3,736 & 3,662 & 0 & 1997 & 4,421 & 4,186 & 0 \\
\hline 1984 & 3,501 & 3,576 & 0 & 1998 & 4,293 & 4,136 & 0 \\
\hline 1985 & 3,463 & 3,647 & 0 & 1999 & 4,258 & 4,239 & 0 \\
\hline 1986 & 3,605 & 3,541 & 0 & 2000 & 4,445 & 4,114 & 0 \\
\hline 1987 & 3,767 & 3,831 & 0 & 2001 & 4,361 & 4,311 & 0 \\
\hline 1988 & 4,046 & 4,019 & 0 & 2002 & 4,401 & 4,066 & 0 \\
\hline 1989 & 4,335 & 4,380 & 1 & 2003 & 4,291 & 3,985 & 0 \\
\hline 1990 & 4,675 & 4,627 & 5 & 2004 & 4,104 & 3,949 & 0 \\
\hline \multirow[t]{2}{*}{1991} & 5,125 & 4,998 & 0 & 2005 & 3,727 & 3,516 & 0 \\
\hline & & & & Total & 117,287 & 114,960 & 6 \\
\hline
\end{tabular}

results for the two contrast breeds will characterize the breeding structure of the Thoroughbred population.

\section{Materials and Methods}

\section{Data}

Registry and pedigree records of foals in Japan from 1978 to 2005, supplied from Equine Research Institute, Japan Racing Association (JRA), were used for the demographic analysis. The numbers of foals by sex in each year are given in Table 1.

\section{Demographic analysis}

\section{(1) Migration rate}

Migration rate is the proportion of genes flowing into the Japanese Thoroughbred population from foreign populations. Migration rate through male parents (male migration rate: $m_{m}$ ) was computed by the proportion of foals produced by imported stallions. Analogously, migration through female parents (female migration rate: $m_{f}$ ) was obtained by the proportion of foals produced by imported mares. Total migration rate $(m)$ was computed as: $m=\frac{m_{m}+m_{f}}{2}$.

\section{(2) Generation interval}

The generation intervals of four gametic pathways, sire to son $\left(L_{m m}\right)$, sire to daughter $\left(L_{m f}\right)$, dam to son $\left(L_{f m}\right)$, and dam to daughter $\left(L_{f f}\right)$, were obtained from records of birth dates of produced foals in each year and the birth dates of their sires and dams. The average generation interval $(L)$ was computed from:

$$
L=\frac{L_{m m}+L_{m f}+L_{f m}+L_{f f}}{4} .
$$

(3) Demographic estimates of effective number of sires and effective population size

Annual effective number of sires $\left(N_{m, e}\right)$ was estimated by the formula given by Nomura [8]:

$N_{m, e}=\frac{N_{m}}{1+C V^{2}}$,

where $N_{m}$ is the number of sires of foals produced in a year, and $C V$ is the coefficient of variation of progeny number of sires. As seen from this formula, $N_{m, e}$ is equal to the actual (census) number of sires $\left(N_{m}\right)$ only when all sires equally contribute to their progeny, i.e., $C V=0$. When there is a variation in the progeny numbers among sires $(C V>0), N_{m, e}$ is always smaller than $N_{m}$.

Following Nomura [8], a demographic estimate of the annual effective population size $\left(N_{e, a}\right)$ was obtained with $N_{m, e}$ as:

$N_{e, a}=4 N_{m, e}$

\section{Results and Discussion}

Figure 1 shows the change in migration rates over the investigated period. After the progressive decline 


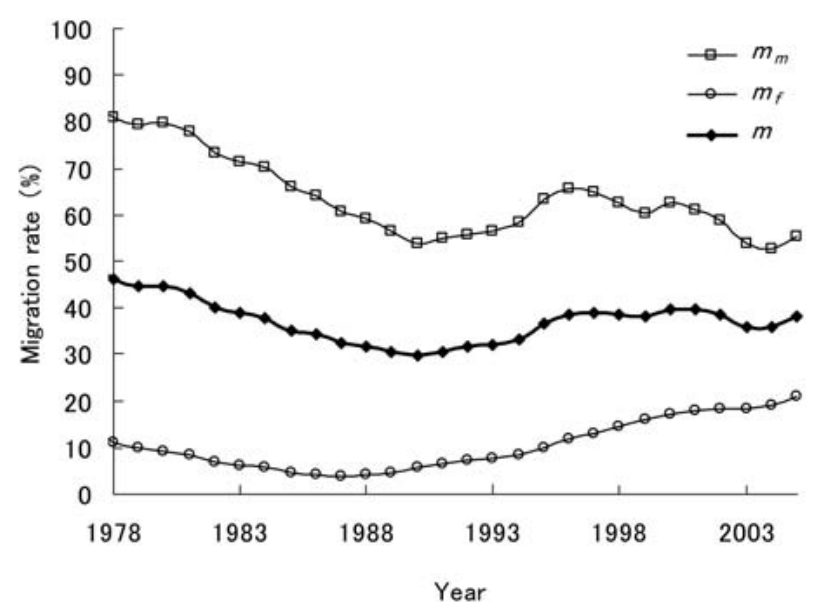

Fig. 1. Changes in total migration rate $(m)$, and migration rates through males $\left(m_{m}\right)$ and females $\left(m_{f}\right)$ in the Japanese Thoroughbred population from 1978 to 2005.

during the period from 1978 to 1990 , the male migration retained the rate of $50-70 \%$. The female migration rate was consistently lower than the male migration rate, but with a gradual increase after 1990. Total migration rate was around $40 \%$ throughout the investigated period, meaning that the amount of annual gene flow from foreign countries into Japan was about $40 \%$.

For a more detail information of migration trend, the migration rates were computed by birth country of migrants. Figures 2 and 3 present the results for male and female migrations, respectively. For male migration, the migration from USA sharply increased during the period from 1990 to 1997. The increase of migration from USA after 1990 was also observed in the female migration. In response to the horse racing boom and the prosperity in 1990s, Japanese horse breeders paid much money for importing foreign breeding stocks with high performance. Several imported horses made great successes as breeding stock in Japan. In particular, progeny of the stallion "Sunday Silence" imported from USA in 1991 won many graded races in Japan. This success would accelerate the migration from USA.

Change of the generation interval is shown in Fig. 4. Although the generation intervals from dams $\left(L_{f m}\right.$ and $\left.L_{f f}\right)$ tended to increase and those from sires $\left(L_{m m}\right.$ and $L_{m f}$ ) showed no consistent trends, the changes in the generation intervals of the four gametic pathways were rather small over the investigated period. The average

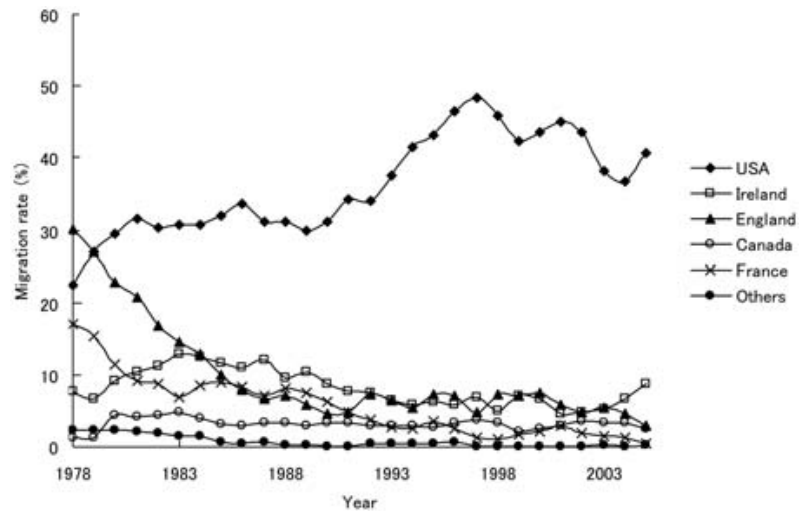

Fig. 2. Changes migration rates through males by their birth country in the Japanese Thoroughbred population from 1978 to 2005 .

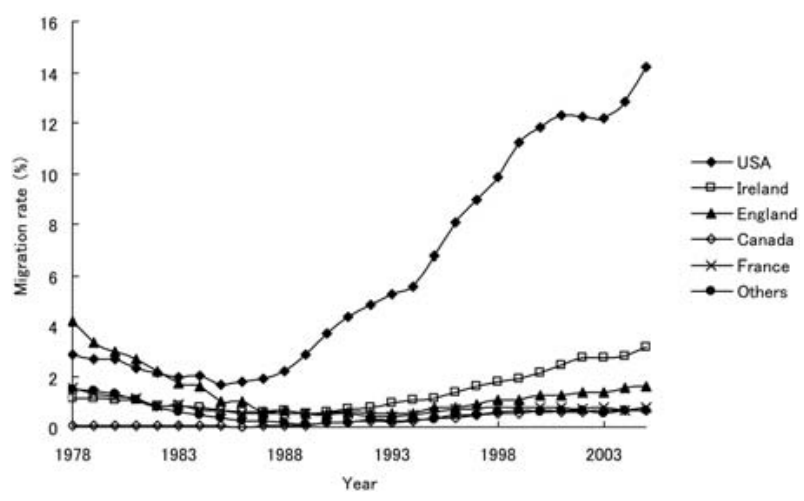

Fig. 3. Changes migration rates through females by their birth country in the Japanese Thoroughbred population from 1978 to 2005 .

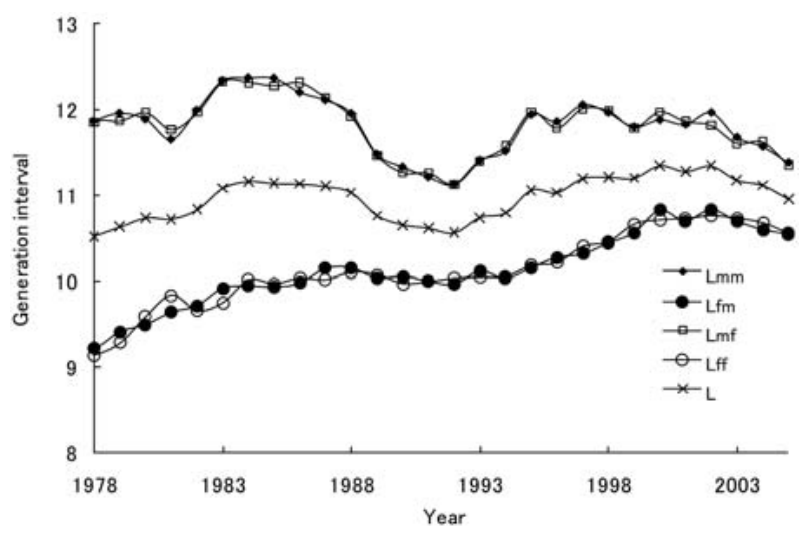

Fig. 4. Changes in generation intervals (years) in the Japanese Thoroughbred population from 1978 to 2005 . $L_{m m}$ : sire to son, $L_{m f}$. sire to daughter, $L_{f m}$ : dam to son, $L_{f f}$ dam to daughter, $L$ : average. 
Table 2. The number of sires $\left(N_{m}\right)$, the coefficient of variation of the foal number of sires $(\mathrm{CV})$, the effective number of sires $\left(N_{m, e}\right)$ and the ratio $N_{m, e} / N_{m}$ in the Japanese Thoroughbred population from 1978 to 2005

\begin{tabular}{lllll}
\hline Year & $N_{m}$ & $C V$ & $N_{m, e}$ & $N_{m, e} / N_{m}$ \\
\hline 1978 & 459 & 0.989 & 232.0 & 0.505 \\
1979 & 452 & 1.018 & 222.0 & 0.491 \\
1980 & 436 & 0.985 & 221.3 & 0.507 \\
1981 & 444 & 0.984 & 225.6 & 0.508 \\
1982 & 431 & 0.942 & 228.3 & 0.530 \\
1983 & 429 & 0.957 & 224.0 & 0.522 \\
1984 & 436 & 1.018 & 214.1 & 0.491 \\
1985 & 430 & 1.007 & 213.5 & 0.496 \\
1986 & 459 & 1.086 & 210.6 & 0.459 \\
1987 & 513 & 1.157 & 219.3 & 0.427 \\
1988 & 561 & 1.211 & 227.3 & 0.405 \\
1989 & 591 & 1.186 & 245.5 & 0.415 \\
1990 & 639 & 1.133 & 279.8 & 0.438 \\
1991 & 680 & 1.165 & 288.4 & 0.424 \\
1992 & 711 & 1.162 & 302.6 & 0.426 \\
1993 & 729 & 1.216 & 294.2 & 0.404 \\
1994 & 714 & 1.303 & 264.6 & 0.371 \\
1995 & 695 & 1.435 & 227.1 & 0.327 \\
1996 & 615 & 1.473 & 194.0 & 0.315 \\
1997 & 560 & 1.532 & 167.3 & 0.299 \\
1998 & 527 & 1.564 & 152.9 & 0.290 \\
1999 & 509 & 1.569 & 147.0 & 0.289 \\
2000 & 488 & 1.632 & 133.2 & 0.273 \\
2001 & 472 & 1.633 & 128.7 & 0.273 \\
2002 & 477 & 1.642 & 129.0 & 0.271 \\
2003 & 477 & 1.669 & 126.0 & 0.264 \\
2004 & 466 & 1.680 & 121.9 & 0.262 \\
2005 & 358 & 1.501 & 110.0 & 0.307 \\
\hline & & & &
\end{tabular}

generation interval was within range of 10.5-11.5 years. Gaffney and Cunningham [3] estimated the average generation interval of Thoroughbred in England as 11 years. Comparable estimates (10.6 and 10.7 years) have been reported in French Thoroughbred [7] and in Brazilian Thoroughbred [11]. Our estimate well agrees with these published estimates. It could be generally concluded that Thoroughbred has a long generation interval from 10 to 12 years compared with other domesticated mammals whose values are half of those or less. As pointed out by Moureaux et al. [7] and Thiruvenkadan et al. [12], the longer generation interval might be due to the fact that Thoroughbred horses begin breeding only after completing their performance in races.

Table 2 shows the number of sires of foals produced in each year $\left(N_{m}\right)$, the coefficient of variation of the progeny numbers of sires $(C V)$, the effective number of

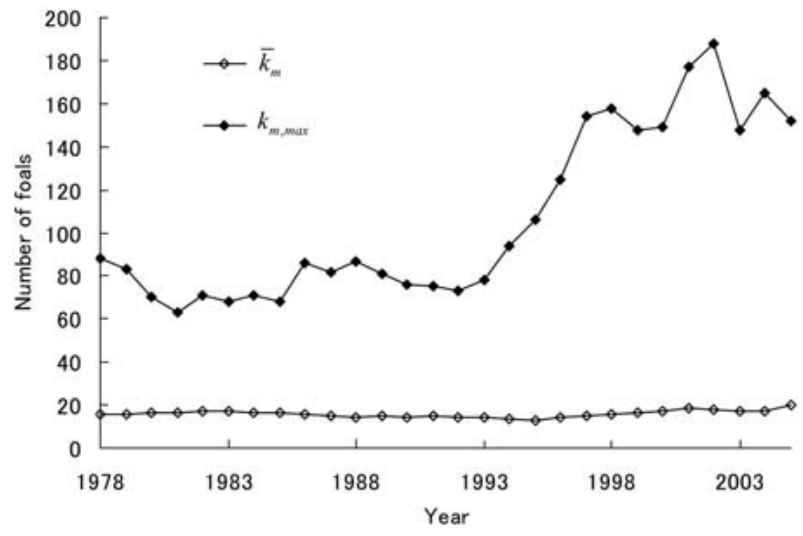

Fig. 5. The average number of foals of sires $\left(\bar{k}_{m}\right)$ and the foal number of the most intensively used sire $\left(k_{\max }\right)$ in the Japanese Thoroughbred population from 1978 to 2005.

sires $\left(N_{m, e}\right)$, and the ratio $N_{m, e} / N_{m}$. After the number of sires $\left(N_{m}\right)$ had been retained the value around 450 until the middle of $1980 \mathrm{~s}$, it tended to increase in the next decade. The increase in $N_{m}$ in this period would be a reflection of the progressively increased importation of stallions particularly from USA (Fig. 2). After the peak of 729 in 1993, $N_{m}$ progressively decreased to 358 in 2005. Although the coefficient of variation of the progeny numbers of sires $(C V)$ was within range of 1.0 1.2 until early $1990 \mathrm{~s}$, it tended to gradually increase and reached the value of 1.6-1.7 in recent years. This increase of $C V$ indicates that the difference of the progeny numbers among sires were enhanced after early 1990s. Both the decreased $N_{m}$ and increased after $C V$ early 1990s lead to a decrease of the effective number of sires $\left(N_{m, e}\right)$. As shown in the fourth column in Table 2, $N_{m, e}$ consistently decreased after the peak of 302.6 in 1992, and reached 120-130 in recent years, which is $25-30 \%$ of the actual number of sires $\left(N_{m}\right)$.

Figure 5 presents the average number of progeny of sires $\left(\bar{k}_{m}\right)$ and the progeny number of the most intensively used sire $\left(k_{\max \text { max }}\right)$ in each year. While the average number $\bar{k}_{m}$ kept the value around 20 over the investigated period, the maximum number $k_{\text {m, } \max }$ sharply increased after 1993. Particularly after 2000, there existed sires with more than 160 foals in a year. The most intensively used sire from 1996 to 2002 was the imported stallion "Sunday Silence". However, the progressively increased $C V$ and decreased $N_{m, e}$ observed in Table 1 were not caused only by this stallion. Although the number of sires having more than 100 foals per year was five in 1995 , the number increased to 
Table 3. The number of sires $\left(N_{m}\right)$, the coefficient of variation of the progeny number of sires $(\mathrm{CV})$, the effective number of sires $\left(N_{m, e}\right)$ and the ratio $N_{m, e} / N_{m}$ in the Japanese Black cattle population from 1978 to 2004

\begin{tabular}{lrrrr}
\hline Year & $N_{m}$ & $C V$ & $N_{m, e}$ & $N_{m, e} / N_{m}$ \\
\hline 1978 & 1,031 & 2.459 & 146.3 & 0.142 \\
1979 & 1,053 & 2.530 & 142.3 & 0.135 \\
1980 & 1,064 & 2.554 & 141.5 & 0.133 \\
1981 & 1,135 & 2.598 & 146.4 & 0.129 \\
1982 & 1,132 & 2.585 & 147.3 & 0.130 \\
1983 & 1,048 & 2.666 & 129.3 & 0.123 \\
1984 & 1,048 & 3.071 & 100.5 & 0.096 \\
1985 & 1,004 & 3.312 & 83.9 & 0.084 \\
1986 & 1,009 & 3.640 & 70.8 & 0.070 \\
1987 & 1,019 & 3.408 & 80.8 & 0.079 \\
1988 & 1,067 & 3.761 & 70.4 & 0.066 \\
1989 & 1,045 & 3.758 & 69.1 & 0.066 \\
1990 & 1,064 & 3.653 & 74.2 & 0.070 \\
1991 & 981 & 3.841 & 62.3 & 0.063 \\
1992 & 966 & 4.146 & 53.1 & 0.055 \\
1993 & 884 & 4.180 & 47.9 & 0.054 \\
1994 & 860 & 4.434 & 41.6 & 0.048 \\
1995 & 834 & 5.007 & 32.0 & 0.038 \\
1996 & 779 & 5.051 & 29.4 & 0.038 \\
1997 & 829 & 5.155 & 29.8 & 0.036 \\
1998 & 787 & 6.032 & 21.1 & 0.027 \\
1999 & 747 & 6.502 & 17.3 & 0.023 \\
2000 & 732 & 7.933 & 11.4 & 0.016 \\
2001 & 719 & 7.237 & 13.5 & 0.019 \\
2002 & 727 & 6.950 & 14.7 & 0.020 \\
2003 & 756 & 7.301 & 13.9 & 0.018 \\
2004 & 752 & 6.917 & 15.4 & 0.020 \\
\hline & & & &
\end{tabular}

15 or more after 2000. This intensive use of a limited number of sires for breeding was the major cause of the increased $C V$ and decreased $N_{m, e}$ in Table 2.

For comparison, Table 3 shows the number of sires $\left(N_{m}\right)$, the coefficient of variation of their progeny number $(C V)$, effective number of sires $\left(N_{m, e}\right)$ and the ratio $N_{m, e} / N_{m}$ in the Japanese Black cattle population (data from [8] with additional recent results). The Japanese Black cattle is the most common beef breed in Japan, with a population of 0.61 million reproductive cows. This breed is characterized by prominent intramuscular fat deposition (marbling). Since liberalization of beef import restriction in 1991, domestic production of high-quality beef has received more emphasis in Japan, and the genetic evaluation of meat characteristics, with the best linear unbiased prediction (BLUP) methodology, was initiated in 1991. Nomura et al. [9, 10] and Honda et al. [5] have shown that the breeding structure of the Japanese Black cattle population had drastically changed after 1991. As shown in Table 3, the number of sires was essentially constant over the period of 1985 to 1990 but showed a gradual decline after 1991. Another notable trend is the sharp increase in the coefficient of variation of progeny number among sires $(C V)$. The value after 2000 was about 3 times larger than in 1985. This increase of $C V$ was mainly due to the intensive use of a few popular sires with high marbling estimated breeding values.

In comparison with the results for Thoroughbred population (Table 2), the most notable feature is the difference of $C V$ between the two populations. As already shown, $C V$ in the Thoroughbred population also increased after early 1990s, but the value was still around 1.7 even after 2000, which is less than $25 \%$ of the corresponding value in the Japanese Black cattle population. This will be a reflection of difference in reproductive methods applied in the two breeds. In the Japanese Black cattle, almost all the progeny are produced by artificial insemination with frozen semen, which enables to reproduce about 60,000 progeny per year from a limited number of sires. In particular, the most intensively used sire produces more than 10,000 progeny in a year. In Thoroughbred, even for the stallion with the maximum number of progeny after 1995, the number of foals in a year was below 200. This is because natural service limits a heavy use of one popular stallion. This difference of reproductive method between the two breeds is also reflected in the effective number of sires. While the actual number of sires $\left(N_{m}\right)$ in the Japanese Black cattle population after 2000 is more than 2 times of that in the Thoroughbred population, the effective number $\left(N_{m, e}\right)$ is below $10 \%$ of $N_{m, e}$ in the Thoroughbred population.

Finally, change in the demographic estimate of the annual effective population size $\left(N_{e, a}\right)$ from 1978 to 2005 is presented in Fig. 6, in which the estimate in the Japanese Black cattle is also shown for comparison. In Thoroughbred, $N_{e, a}$ declined gradually after the peak over 1,000 in 1992. However, the effective size after 2000 still retained the value around 500, which is 10 times of the corresponding estimates in the Japanese Black cattle.

In conclusion, our demographic analysis clearly showed that the breeding structure of the Japanese Thoroughbred population has been largely changed after early 1990s. This change could be considered as a reflection of the horse racing boom and the prosperity at that time. Breeders imported many horses from USA as breeding stock. In accordance, limited stallions with 


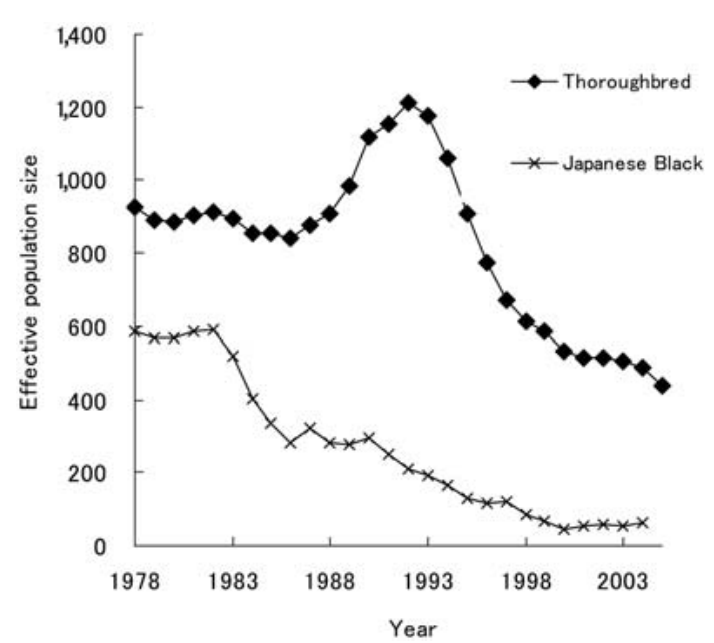

Fig. 6. Demographic estimates of the annual effective size of Japanese Thoroughbred and Japanese Black cattle populations.

high performance have been intensively used for breeding, leading to the declines of the effective number of sires and the effective population size. However, the magnitude of the change was much smaller than the similar change in the Japanese Black cattle population. The smaller change in the Thoroughbred population will ascribe to the fact that the reproduction is strictly limited to natural service, which prevents breeders from using a few prominent stallions heavily. In succeeding reports, we assess the impact of the change in breeding structure on the genetic constitution of population and evaluate the genetic diversity by population genetic analysis with pedigree information.

\section{References}

1. Cunningham, E.P. 1991. The genetics of Thoroughbred horses. Sci. Amer. 264: 56-62.

2. Cunningham, E.P., Dooley, J.J., Splan, R.K., and
Bradley, D.G. 2001. Microsatellite diversity, pedigree relatedness and the contributions of founder lineages to thoroughbred horses. Anim. Genet. 32: 360-364.

3. Gaffiney, B., and Cunningham, E.P. 1988. Estimation of genetic trend in racing performance of Thoroughbred horses. Nature 332: 722-723.

4. Hill, W.G. 1988. Why aren't horses faster? Nature 332: 678.

5. Honda, T., Nomura, T., Yamaguchi, Y., and Mukai, F. 2002. Pedigree analysis of genetic subdivision in a population of Japanese Black cattle. Anim. Sci. J. 73: 445-452.

6. Honda, T., Nomura, T., Yamaguchi, Y., and Mukai, F. 2004. Monitoring of genetic diversity in the Japanese Black cattle population by the use of pedigree information. J. Anim. Breed. Genet. 121: 242-252.

7. Moureaux, S., Verrier, E., Ricard, A., and Meriaux, J.C. 1996. Genetic variability within French race and riding horse breeds from genealogical data and blood marker polymorphisms. Genet. Sel. Evol. 28: 83-102.

8. Nomura, T. 2002. Effective size of populations with unequal sex ratio and variation in mating success. J. Anim. Breed. Genet. 118: 297-310.

9. Nomura, T., Honda, T., and Mukai, F. 2001. Inbreeding and effective population size of Japanese Black cattle. J. Anim. Sci. 79: 366-370.

10. Nomura, T., Honda, T., and Mukai, F. 2005. Monitoring and preservation of genetic diversity in livestock breeds: a case study of the Japanese Black cattle population. Current Topics Genet. 1: 5971.

11. Taveira, R.Z., Mota, M.D.S., and Oliveira, H.N. 2004. Population parameter in Brazilian Thoroughbred.J. Anim. Breed. Genet. 121: 384-391.

12. Thiruvenkadan, A.K., Kandasamy, N., and Panneerselvam, S. 2009. Inheritance of racing performance of Thoroughbred horses. Livest. Sci. 121: 308-326. 